\title{
Multi-messenger Observations of a Binary Neutron Star Merger
}

\author{
Rosa Poggiani* ${ }^{\dagger}$ on behalf of the LIGO Scientific Collaboration and the Virgo \\ Collaboration \\ Università di Pisa and Istituto Nazionale di Fisica Nucleare, Sezione di Pisa \\ E-mail: rosa.poggiani@unipi.it
}

On 2017 August 17 the Advanced LIGO and Advanced Virgo interferometers observed the merger of a binary neutron star system. The INTEGRAL SPI-ACS and Fermi-GBM instruments independently observed the short Gamma Ray Burst GRB 170817A, after a delay of about $1.7 \mathrm{~s}$. GW170817 was localized within a sky region of about $30 \mathrm{deg}^{2}$ at a luminosity distance of $40 \mathrm{Mpc}$. A worldwide electromagnetic follow-up campaign detected an optical counterpart (SSS17a/AT 2017gfo) in the elliptical galaxy NGC 4993. The optical and infrared spectroscopic observations during the first days after the merger showed the signature of a kilonova. The first X-ray and radio detections of the afterglow occurred at 9 and 16 days after the merger, respectively. The multi-messenger observations suggest that GW170817 was produced by the merger of two neutron stars in the galaxy NGC 4993, followed by the short gamma ray burst GRB 170817A and by a kilonova powered by the radioactive decay of r-process nuclei synthesized in the ejecta. This review is an update of [110], summarizing the multi-messenger observations of GW170817 from the discovery to the late stages.

Multifrequency Behaviour of High Energy Cosmic Sources - XIII - MULTIF2019

3-8 June 2019

Palermo, Italy

\footnotetext{
* Speaker.

${ }^{\dagger}$ Corresponding author
} 


\section{Introduction}

The Advanced LIGO and Advanced Virgo detectors completed the $\mathrm{O} 2$ observing run in August 2017. The majority of detected gravitational events during the O1, O2 runs are mergers of binary black holes systems: GW159014 [1], GW151226 [2], GW170104 [3], GW170608 [4], GW170814 [5] and the events in the GWTC-1 catalog [20].

On 2017 August 17 at 12:41:04 UTC the Advanced LIGO and Advanced Virgo interferometers observed GW170817, the first gravitational merger of a binary system of neutron stars [6]. The Fermi Gamma-ray Burst Monitor (GBM) and the INTEGRAL spectrometer anticoincidence shield (SPI-ACS) independently detected the short Gamma Ray Burst GRB 170817A after a delay of 1.7 s [65], [118], [8]. The merger GW170817 and the Gamma Ray Burst GRB 170817A were followed by the ultraviolet, optical and near infrared emission and, later, by X-ray and radio emission [7].

The joint observation of a gravitational merger and of its electromagnetic counterpart suggests that binary neutron star systems are the progenitors of short GRBs or at least part of them, which are expected to show a long lasting afterglow from the radio to the X-rays. The multi-messenger observations [7] suggest that binary neutron star mergers are sites of r-process nucleosynthesis of heavy elements whose decay produce a kilonova [105]. [59], [106], [86], [79], [101], [40], [41], [125], [42], [54], [96]. The GW170817 event has constrained neutron star properties [6], [15], [13], [16], [20], [19] and has allowed a determination of the Hubble constant independent from the electromagnetic observations [9]. This review summarizes the multi-messenger observations of GW170817/GRB 170817A from the early to the late stages, together with their impact on astrophysics and fundamental physics.

\section{Gravitational Observation of GW170817}

On August 17, 2017 at 12:41:04 UTC the Advanced LIGO and Advanced Virgo interferometers observed the event GW170817 with a combined signal-to-noise ratio of 32.4 and an estimated false alarm rate below one per $8.0 \times 10^{4}$ years [6]. The time freqeuncy map of the signal is shown in Fig. 1. The event source was localized within a sky region of about $28 \mathrm{deg}^{2}$ at a luminosity distance of $40_{-14}^{+8} \mathrm{Mpc}[6]$.

The estimated total mass is in the range from 2.72 to $3.29 \mathrm{M}_{\odot}$, where individual masses range from 0.86 to $2.26 \mathrm{M}_{\odot}$ [6]. The primary and secondary masses have been estimated using both low-spin and high spin priors: for the former, masses are in the ranges 1.36-1.60 $\mathrm{M}_{\odot}$ and 1.17$1.36 \mathrm{M}_{\odot}$, for the latter in the range 1.36-2.26 $\mathrm{M}_{\odot}$ and 0.86-1.36 $\mathrm{M}_{\odot}$ [6]. Both estimations agree with the known masses of neutron stars in binary systems, but are not consistent with the masses of black holes in galactic X-ray binary systems. The observation of the GW170817 merger has constrained different properties of neutron stars, among them their tidal deformability, radii, equations of state [6], [15], [13], [16], [20], [19]. The merger remnant [12], [17] could be a short lived supramassive/hypermassive neutron star or a stable neutron star or a black hole formed in a collapse. A neutron star is expected to radiate gravitational waves with short $(\leq 1 \mathrm{~s})$ or intermediate $(\leq 500 \mathrm{~s})$ duration, while a black hole is expected to emit $\mathrm{kHz}$ gravitational waves during the ringdown phase. All searches, for short and intermediate duration 1-4 kHz signals [12] and for longer duration signals [17], have been negative. 


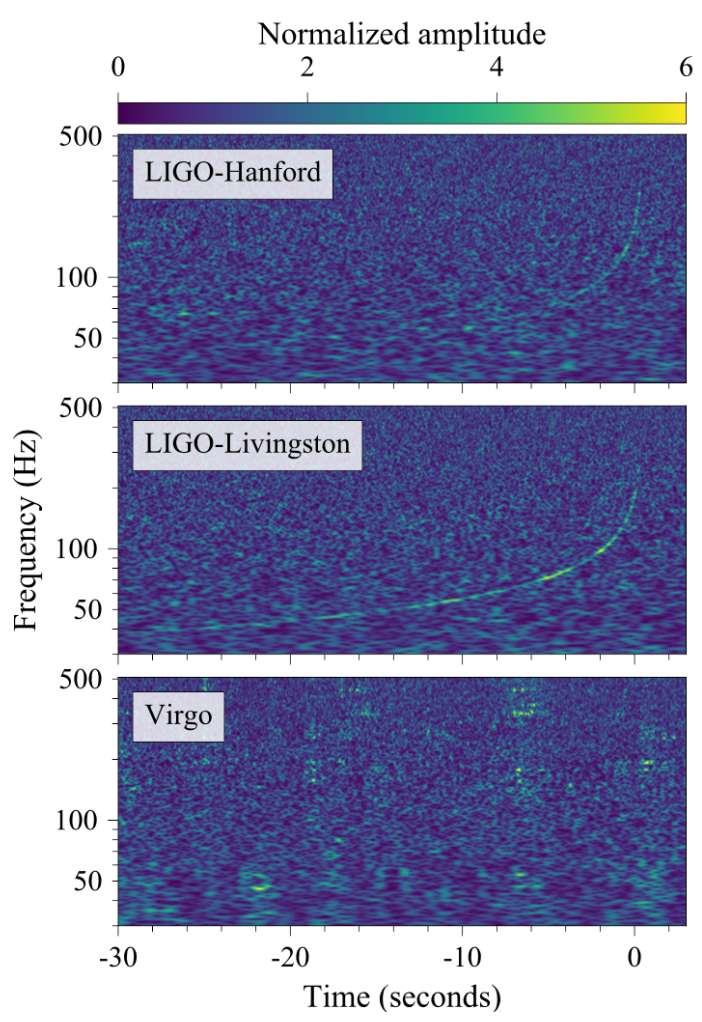

Figure 1: Time-frequency maps of the GW170817 signal detected in the LIGO-Hanford, LIGO-Livingston and Virgo detectors; adapted from [6]

The observation of GW170817 has allowed to update the local coalescence rate of binary neutron star systems, $1540_{-1220}^{+3200} \mathrm{Gpc}^{-3} \mathrm{yr}^{-1}$ [6] and the contribution of unresolved distant systems to the stochastic gravitational wave background [14]. Binary neutron star mergers are expected to show electromagnetic emission from dynamica ejecta. The ejecta mass distribution ranges from $10^{-3}$ to $10^{-2} \mathrm{M}_{\odot}$ [10], that in combination with the binary neutron star merger rate [6], can explain the abundance of Galactic r-elements if about $10 \%$ of the ejected matter is converted to r-process elements [10], whose observations are described below.

The GW170817 event has been used for various tests of General Relativity and of strong field dynamics [18]: deviations from General Relativity during the Post Newtonian inspiral phase; violation of the Local Lorentz Invariance; anomalous dispersion during propagation; number of large extra spatial dimensions; alternative polarization states.

\section{Early High Energy Observations of the Counterpart}

The Fermi-GBM instrument observed the short Gamma Ray Burst GRB 170817A, on 2017 August 17 at 12:41:06 UTC [65], [8], while the INTEGRAL instrument detected the event in an off-line analysis triggered by the LIGO-Virgo alert [118], [8]. The gravitational and gamma ray signals, reported in Fig. 2, showed a difference in the arrival times of $1.734 \pm 0.054 \mathrm{~s}$ [65], [8]. 


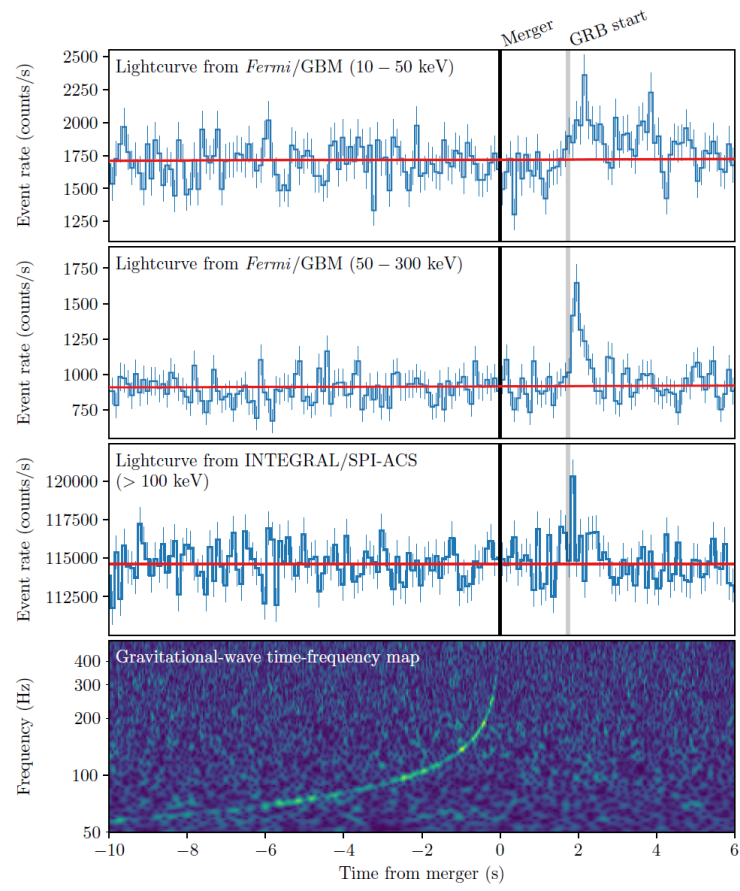

Figure 2: Joint detection of GW170817 and the short Gamma Ray Burst GRB 170817A. From top to bottom: Fermi-GBM light curve of GRB 170817A (10-50 keV); as above (50-300 keV); INTEGRAL SPIACS light curve (above $100 \mathrm{keV}$ ); time-frequency map of GW170817 gravitational signal; adapted from [8]

The duration of GRB 170817A is $\mathrm{T}_{90}=2.0 \pm 0.55 \mathrm{~s}$, consistent with the classification as a short Gamma Ray Burst [65], [7]. The burst had two components, a Comptonized spectrum and a tail with a soft black body spectrum [65]. GRB 170817A is fainter than the typical short Gamma Ray Bursts by some orders of magnitude [8]. The early observations have been modelled either wih an isotropic fireball expansion [116] or various jet models: a uniform jet observed at large angles, a mildly relativistic isotropic cocoon with a chocked jet, a structured jet [109], [76], [74], [128], [83], [8],

No gamma ray excess was detected during the first days after the merger by AGILE (above $30 \mathrm{MeV}$ ) [134], ASTROSAT (30-200 keV) [39], CALET (above $1 \mathrm{GeV}$ ) [26], Fermi-LAT (0.1-1 $\mathrm{GeV}$ ) [28], HAWC (above $40 \mathrm{TeV}$ ) [94], HESS (270 GeV-8.55 TeV) [22], [23], Insight-HXMT (0.2-5 MeV) [87].

The observation of GW170817 with three interferometers localized GW170817 within about $30 \mathrm{deg}^{2}$, narrowing the region for the search of the electromagnetic counterpart [7] (Fig. 3). The candidate host galaxies within the gravitational error box were 54 [49].

\section{Early Ultraviolet, Optical and Infrared Observations}

On August 17, 2017 at 23:33 UTC (10.87 hours after the merger) the One-Meter, TwoHemisphere (1M2H) Collaboration discovered a new optical transient, SSS17a/AT 2017gfo, in the elliptical galaxy NGC 4993, using the 1m Swope telescope, Las Campanas, Chile [51], [121]. 


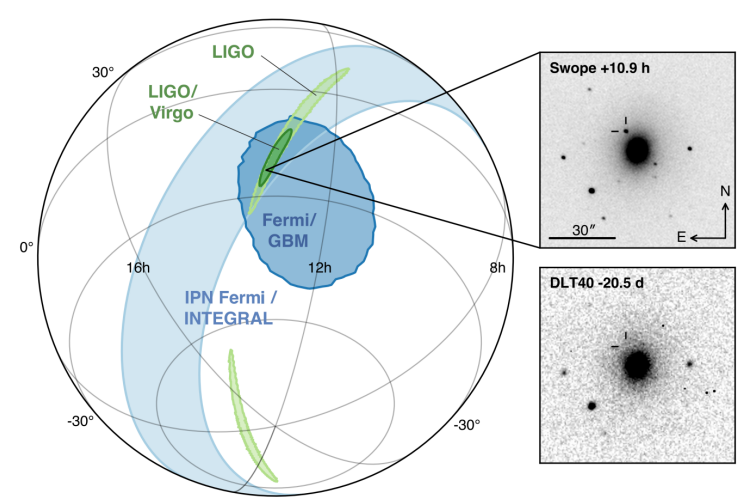

Figure 3: Sky localization of the gravitational, gamma-ray and optical signals of GW170817/GRB 170817A. Left: LIGO (light green), LIGO-Virgo (dark green), Fermi and INTEGRAL (light blue), Fermi GBM (dark blue) localization. Right: localization of the host galaxy NGC 4993 by 1M2H Collaboration at 10.9 hours after the merger and the DLT40 pre-discovery image; adapted from [7]

The transient was independently detected by DLT40 at $11.08 \mathrm{hr}$ after the event [133], VISTA at $11.24 \mathrm{hr}$ [126], MASTER at $11.31 \mathrm{hr}$ [88], DECam at $11.40 \mathrm{hr}$ [123], Las Cumbres at $11.57 \mathrm{hr}$ [34]. The new transient was not present in DLT40 archival images secured a few weeks before the merger [133]. The merger was localized at a projected distance of $2 \mathrm{kpc}$ from NGC 4993 center [11]; the progenitor has been discussed by [11]. The known distance of NGC 4993 was consistent with the gravitational luminosity distance. A worldwide network of ground and space based observatories participated to the electromagnetic follow-up of SSS17a/AT 2017gfo, that was associated to GW170817 [7].

The photometric light curves of GW170817/GRB 170817A/AT 2017gfo during the first weeks after the merger are reported in Fig. 4, left. Optical and infrared photometric observations have been performed by J-GEM [132], DLT-40 [137], Las Cumbres [35], RATIR [66], TOROS [56], Australian facilities [33], SALT [45], Subaru [127]. A compilation of photometric data during the early stage has been presented by [36].

The optical and infrared spectra evolved from an initial featureless blue black body spectrum $(11000 \mathrm{~K})$ through a dimming of the blue component to the emergence of broad lanthanide features [120], [45], [95], [109], [89], [103] [74], [73], [77], [35], [53], [122], [58], [128], [85], [126], [48]. The evolution could be explained by the kilonova model [105]. [59], [106], [86], [79], [101], [40], [41], [125], [42], [54], [96]. The kilonova probably included three components [135]: a fast lanthanide poor component, an intermediate opacity one and a slower lanthanide rich component. Some spectra secured during the first days after the merger are reported in Fig. 4, right. Polarimetric observations suggested a symmetric geometry of the emitting region [52].

The environment of the host galaxy NGC 4993 has been investigated after the merger [107], [72], [85], [62], [43]. The distance of NGC 4993 has been measured after GW170817 using four different methods: heliocentric redshift and Fundamental Plane of galaxies (combined 41.0 \pm 3.1 Mpc) [71], fundamental plane (37.7 $\pm 8.7 \mathrm{Mpc}$ ) [72], surface brightness fluctuations (40.7 $\pm 1.4 \pm 1.9$ Mpc) [46], Globular Cluster Luminosity Function (41.65 $\pm 3.00 \mathrm{Mpc}$ ) [84].

The inclination of the system, initially constrained to be smaller than 55 degrees [6], was later 

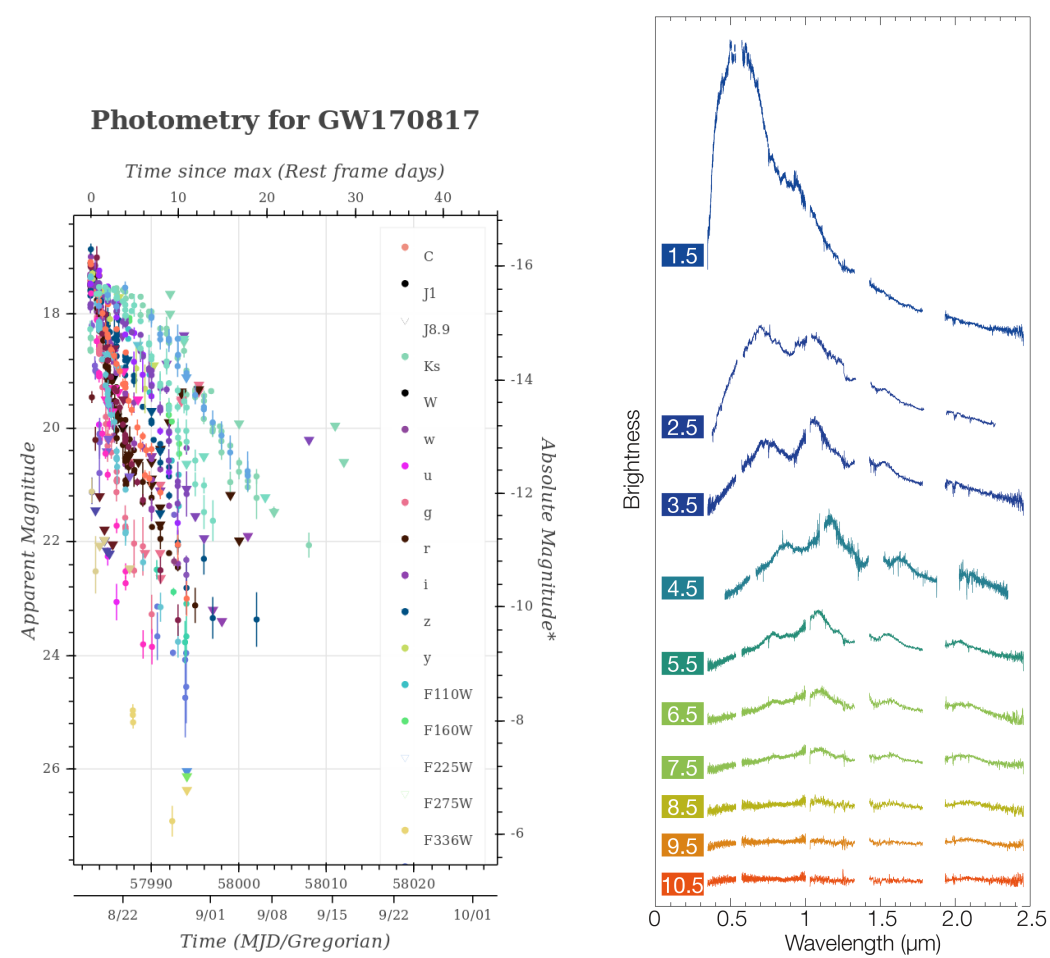

Figure 4: Left: Light curve of GW170817 in different photometric bands during the first month after the merger, image credits: https://kilonova.space/kne/GW170817/. Right: spectra of GW170817 in the first days after the merger, image credits: ESO/E. Pian et al./S. Smartt \& ePESSTO)

constrained by the redshift of NGC 4993 and the Hubble constant measurement by the Dark Energy Survey, to be smaller than 28 degrees [91].

\section{Early X-ray and Radio Observations}

The early X-ray observations of GW170817 by MAXI (2-10 keV) at +0.19 days [124], Chandra at +2.2 d [92], INTEGRAL JEM-X at +6 d [118], Swift and NuSTAR [60] failed to detect any counterpart. The X-ray afterglow was firstly detected at 9 days after the merger (2017 Aug 26) by the Chandra Observatory [128]. The flux increased in the following days [128], [68].

The radio afterglow of GW170817 was firstly detected 16 days after the merger with VLA (2017 September 2) [70]. The afterglow was observed at $+19 \mathrm{~d}$ by ATCA [70] and VLA [31], [70], at $+39 \mathrm{~d}$ with VLA [31], from +1.4 to $+44 \mathrm{~d}$ with ALMA, GMRT [78]. The radio flux showed an increase during the following weeks [70], [97]. Limits on the magnetic field of the shocked ejecta has been set with polarimetric observations [50].

\section{Early Neutrino Observations}

Different observatories have searched for neutrino emission mainly in two time windows, within $\pm 500 \mathrm{~s}$ around the merger epoch and during 14 days after the merger. The searches span a broad range of neutrino energies, from $\mathrm{MeV}$ to PeV. The ANTARES, IceCube, and Pierre Auger 
Observatories searched for high-energy neutrinos from GW170817 in the GeV-EeV energy range [29]. The Super-Kamiokande Observatory searched for neutrino events in the energy range from 3.5 MeV to about $100 \mathrm{PeV}$ [24]. The Baikal-GVD telescope searched for high energy neutrinos, from $\mathrm{TeV}$ to $100 \mathrm{PeV}$ [37]. The Baksan Underground Scintillation Telescope searched for neutrinos and antineutrinos above $1 \mathrm{GeV}$ [108]. The LVD detector searched for an excess of events with an energy above $5 \mathrm{MeV}$ during a time window of $3000 \mathrm{~s}$ [27]. All neutrino searches have been negative.

\section{Late Multiwavelength Observations}

The early multiwavelength observations of GW170817 has been used to model the prompt high energy emission [112]. The multiwavelength monitoring of GW170817 resumed after the source emerged from behind the Sun and is still ongoing. X-ray observations were secured with Chandra at $+108,+111 \mathrm{~d}$ [111] and at $+109 \mathrm{~d}$ [115]. Radio observatios were secured up to $+107 \mathrm{~d}$ with VLA, ATCA, GMRT [97]. Additional Chandra (+153, +163 d), HST (+137 d) and VLA (+80 d) observations were secured by [93]. The optical afterglow was observed by [89] at $+109 \mathrm{~d}$ after the merger. Radio observations from 7 to 152 days after the merger were made by [113]. Infrared observations of GW170817 at $+43 \mathrm{~d}$ and $+74 \mathrm{~d}$ detected the object at $4.5 \mu \mathrm{m}$, but not at $2.6 \mu \mathrm{m}$ [136], [75].

The initial brightening in the radio and X-rays was followed by a turnover and by a later decline. The XMM-Newton X-ray observations by [55] at $+135 \mathrm{~d}$ and the VLA, ATCA radio observations by [57] from $+125 \mathrm{~d}$ to $+200 \mathrm{~d}$ showed the afterglow fading, suggesting a peak at $149 \pm 2$ $\mathrm{d}$ after the merger [57]. The rise and decline were confirmed by observations with Chandra (from $+160 \mathrm{~d}$ to +260 d) [104], VLA, ATCA, GMRT, MeerKat up to +300 d [98], Chandra and ATCA up to $+391 \mathrm{~d}$ [129], LOFAR in the intervals 130-138 d and 371-374 d [44]. VLBI observations were secured from $+7 \mathrm{~d}$ to $+230 \mathrm{~d}$ [99] and at $+207 \mathrm{~d}$ by [64]. The optical afterglow was observed with HST from $+110 \mathrm{~d}$ to $+584 \mathrm{~d}$ after the merger [80], [63]. Chandra and VLA observations of GW170817 from $+521 \mathrm{~d}$ to $+743 \mathrm{~d}$ after the merger showed a late non thermal emission [69]. Chandra observations between $+935 \mathrm{~d}$ and $+942 \mathrm{~d}$ confirmed the decline in the X-rays, with a negative detection in the radio domain [131].

The panchromatic light curve from +0.5 to $+940 \mathrm{~d}$ after the merger, including optical, radio and X-ray data, has been presented by [90]. The afterglow light curve increased as $\mathrm{t}^{0.86}$ up to about 155 days after the merger and later declined as $\mathrm{t}^{-1.90}$ [90]. The afterglow was optically thin during its evolution, with a spectral index of -0.569 [90]. The panchromatic afterglow light curve is shown in Fig. 5.

The rising light curve of the afterglow ruled out an on axis jet [60], [70], [30], [128], [92], [68], [78]. [100], [115], [89], [113], [83]. A mildly relativistic wide angle outflow from a cocoon was suggested by [97], [82], [67]. Also the afterglow decline after the peak ruled out the isotropic model, but could not assess whether the jet was chocked or not [50], [80], [93], [31], since an initial rise, a peak and a decay are expected for both of them [102]. The VLBI observations by [99] showed a proper motion of the source by 2.7 mas from $+75 \mathrm{~d}$ to $+230 \mathrm{~d}$ and suggested that the jet core had an opening angle smaller than a few degrees. The late observations suggest that GW170817 produced an off axis structured relativistic jet [98], [130], [63], [64], [69], [80]. 


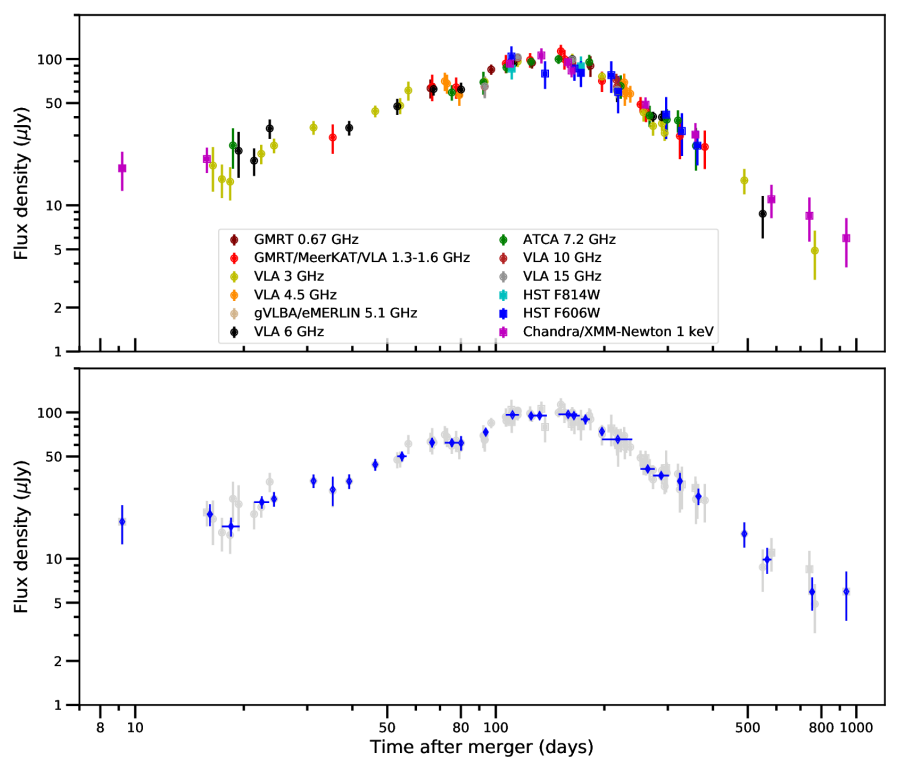

Figure 5: Upper panel: the panchromatic (optical, radio and X-ray) afterglow light curve of GW170817, from $+0.5 \mathrm{~d}$ to $+940 \mathrm{~d}$ after the merger. Lower panel: averaged light curve (blue data points). Credits: http://www.tauceti.caltech.edu/kunal/gw170817/

\section{Cosmology with Gravitational Waves}

The observation of an electromagnetic counterpart of GW170817 has been instrumental to perform cosmology with gravitational wave observations. The delay of gamma rays with respect to the gravitational emission has constrained the fractional difference between the speed of light and the speed of gravity at the level of $10^{-15}-10^{-16}$ [8]. Some classes of modified gravity or dark energy models [32], [38], [81], [117] have been constrained or ruled out.

GW170817 is the first detected standard siren, the gravitational counterpart of the standard candle [119]. The combination of the gravitational distance and of the velocity of the optical counterpart allows the direct measurement of the Hubble constant $\mathrm{H}_{0}$ [119]. To date, there is tension between the value of the Hubble constant measured using CMB observations, $67.8 \pm 0.9 \mathrm{~km}$ $\mathrm{s}^{-1} \mathrm{Mpc}^{-1}$ [25] and the value measured with the Cepheid variables, $73.48 \pm 1.66 \mathrm{~km} \mathrm{~s}^{-1} \mathrm{Mpc}^{-1}$ [114]. The luminosity distance of GW170817 combined with the recession velocity of the host galaxy NGC 4993 [9] has produced an independent estimation of the Hubble constant, $70_{-8}^{+12} \mathrm{~km} \mathrm{~s}$ $\mathrm{Mpc}^{-1}$ (Fig. 6).

The precision on the Hubble constant is expected to improve at the level of $2 \%$ in five years and $1 \%$ in ten years with the detection of new binary neutron star mergers [47]. The Hubble constant can be estimated also for events without electromagnetic counterpart using statistical methods involvng the galaxies contained in the merger localization region [61], [21].

\section{Conclusions}

The worldwide observation campaign of GW170817 with gravitational waves, all electromagnetic probes and neutrinos has started multi-messenger astronomy. The joint observations of 


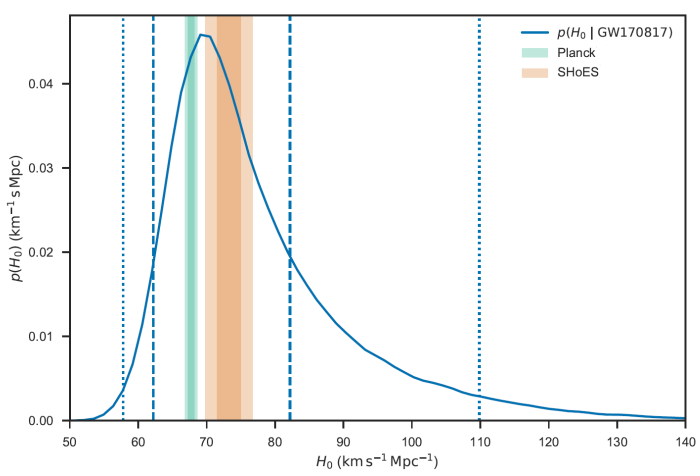

Figure 6: Relative probability of different values of Hubble constant estimated by [9] (solid blue curve), limits of $68.3 \%$ and $95.4 \%$ credible intervals (dashed and dotted blue vertical lines); range of values inferred from CMB data, Planck satellite (green band); range of values inferred from the SHoES analysis of Cepheids and type Ia supernovae (orange band). Adapted from [9]

GW170817 and of the short Gamma Ray Burst GRB 170817A suggests that at least some sGRB are associated to binary neutron star mergers and has constrained the fractional difference between the speed of gravity and the light speed. The electromagnetic follow-up of the kilonova showed lanthanide rich ejecta. The GW170817 merger has constrained neutron stars properties and has produced the first independent estimate of the Hubble constant outside the electromagnetic domain.

\section{References}

[1] B. P. Abbott et al., PRL 116 (2016) 061102.

[2] B. P. Abbott et al., PRL 116 (2016) 241103.

[3] B. P. Abbott et al., PRL 118 (2017) 221101.

[4] B. P. Abbott et al., ApJ 851 (2017) L35.

[5] B. P. Abbott et al., PRL 119 (2017) 141101.

[6] B. P. Abbott et al., PRL 119 (2017) 161101.

[7] B. P. Abbott et al., ApJL 848 (2017) L12.

[8] B. P. Abbott et al., ApJL 848 (2017) L13.

[9] B. P. Abbott et al., Nat 551 (2017) 85.

[10] B. P. Abbott et al., ApJ 850 (2017) L39.

[11] B. P. Abbott et al., ApJ 850 (2017) L40.

[12] B. P. Abbott et al., ApJ 851 (2017) L16.

[13] B. P. Abbott et al., PRL 121 (2018) 161101.

[14] B. P. Abbott et al., PRL 120 (2018) 091101.

[15] B. P. Abbott et al., PRX 9 (2019) 011001. 
[16] B. P. Abbott et al., PRL 122 (2019) 061104.

[17] B. P. Abbott et al., ApJ 875 (2019) 160.

[18] B. P. Abbott et al., PRL 123 (2019) 011102.

[19] B. P. Abbott et al., CQG 37 (2020) 045006.

[20] B. P. Abbott et al., PRX 9 (2019) 031040.

[21] B. P. Abbott et al., arXiv:1908.06060.

[22] H. Abdalla et al., ApJ 850 (2017) 22.

[23] H. Abdalla et al., ApJL 894 (2020) L16.

[24] K. Abe et al., ApJ 857 (2018) L4.

[25] P. A. R. Ade et al., A\&A 594 (2016) A13.

[26] O. Adriani et al., ApJ 863 (2018) 160.

[27] N. Y. Agafonova et al., J. Phys.: Conf. Ser. 1390 (2019) 012088.

[28] N. Ajello et al., ApJ 861 (2018) 85.

[29] A. Albert et al., ApJ 850 (2017) L35.

[30] K. D. Alexander et al., ApJL 848 (2017) L21.

[31] K. D. Alexander et al., ApJL 863 (2018) L18.

[32] L. Amendola et al., PRL 120 (2018) 131101.

[33] I. Andreoni et al., PASA 34 (2017) 169.

[34] I. Arcavi et al., Nat 551 (2017) 64.

[35] I. Arcavi et al., ApJL 848 (2017) L33.

[36] I. Arcavi et al., ApJ 855 (2018) L23.

[37] A. D. Avrorin et al., JETPL 108 (2018) 787.

[38] T. Baker et al., PRL 119 (2017) 251301.

[39] A. Balasubramanian et al., GCN 21514 (2017).

[40] J. Barnes and D. Kasen, ApJ 775 (2013) 18.

[41] E. Berger et al., ApJL 774 (2013) L13.

[42] E. Berger, ARA\&A 52 (2014) 43.

[43] P. K. Blanchard et al., ApJ 848 (2017) L22.

[44] J. W. Broderick et al., MNRAS 494 (2020) 5110.

[45] D. A. H. Buckley et al., MNRAS 474 (2018) L71.

[46] M. Cantiello et al., ApJ 584 (2018) L31.

[47] H.-Y. Chen et al., Nat 562 (2018) 545.

[48] R. Chornock et al., ApJ 848 (2017) L19.

[49] D. O. Cook et al., GCN 21519 (2017). 
[50] A. Corsi et al., ApJ 861 (2018) L10.

[51] D. A. Coulter et al., Sci 358 (2017) 1556.

[52] S. Covino et al., NatAs 1 (2017) 791.

[53] P. S. Cowperthwaite et al., ApJ 848 (2017) L17.

[54] P. D’Avanzo, JHEAp 7 (2015) 73.

[55] P. D’Avanzo et al., A\&A 613 (2018) L1.

[56] M. C. Diaz et al., ApJL 848 (2017) L29.

[57] D. Dobie et al., ApJ 858 (2018) L15.

[58] M. R. Drout et al., Sci 358 (2017) 1570.

[59] D. Eichler et al., Nat 340 (1989) 126.

[60] P. A. Evans et al., Sci 358 (2017) 1565.

[61] M. Fishbach et al., ApJ 871 (2019) L13.

[62] W. Fong et al., ApJ 848 (2017) L23.

[63] W. Fong et al., ApJL 883 (2019) L1.

[64] G. Ghirlanda et al., Sci 363 (2019) 968.

[65] A. Goldstein et al., ApJ 848 (2017) L14.

[66] V. Z. Golkhou et al., ApJ 857 (2018) 81.

[67] O. Gottlieb et al., MNRAS 473 (2018) 576.

[68] D. Haggard et al., ApJL 848 (2017) L25.

[69] A Hajela et al., ApJ 886 (2019) L17.

[70] G. Hallinan et al., Sci 358 (2017) 1579.

[71] J. Hjorth et al., ApJL 848 (2017) L31.

[72] M. Im et al., ApJL 849 (2017) L16.

[73] D. Kasen et al., Nat 551 (2017) 80.

[74] M. M. Kasliwal et al., Sci 358 (2017) 6370.

[75] M. M. Kasliwal et al., arXiv:1812.08708 (2019).

[76] A. Kathirgamaraju et al., MNRAS 473 (2018) L121.

[77] C. D. Kilpatrick et al., Sci 358 (2017) 1583.

[78] S. Kim et al., ApJ 850 (2017) 850.

[79] S. Kulkarni, arXiv: astro-ph/0510256 (2005).

[80] G. P. Lamb et al., ApJ 870 (2019) L15.

[81] D. Langlois et al., PRD 97 (2018) 061501.

[82] D. Lazzati et al., ApJL 848 (2017) L6.

[83] D. Lazzati et al., PRL 120 (2018) 241103. 
[84] M. G. Lee et al., ApJ 859 (2018) L6.

[85] A. J. Levan et al., ApJL 848 (2017) L28.

[86] L.-Z. Li and B. Paczynski, ApJ 507 (1998) 59.

[87] T. Li et al., SCPMA 61 (2018) 31011.

[88] V. M. Lipunov et al., ApJL 850 (2017) L18.

[89] J. D. Lyman et al., NatAs 2 (2018) 751.

[90] S. Makhathini et al., arXiv:2006.02382.

[91] I. Mandel, ApJ 853 (2017) L12.

[92] R. Margutti et al., ApJL 848 (2017) L20.

[93] R. Margutti et al., ApJ 856 (2018) L18.

[94] I. Martinez-Castellanos et al., GCN 21683 (2017).

[95] C. McCully et al., ApJL 848 (2017) L32.

[96] B. D. Metzger, LRR 20 (2017) 3.

[97] K. P. Mooley et al., Nat 554 (2018) 207.

[98] K. P. Mooley et al., ApJ 868 (2018) L11.

[99] K. P. Mooley et al., Nat 561 (2018) 355.

[100] A. Murguia-Berthier et al., ApJL 848 (2017) L34.

[101] E. Nakar, PhR 442 (2007) 166.

[102] E. Nakar and T. Piran, MNRAS 478 (2018) 407.

[103] M. Nicholl et al., ApJ 848 (2017) L18.

[104] M. Nynka et al., ApJL 862 L19. (2018).

[105] B. Paczynski, ApJL 308 (1986) L43.

[106] B. Paczynski, AcA 41 (1991) 257.

[107] Y.-C. Pan et al., ApJL 848 (2017) L30.

[108] V. B. Petkov et al., JETP Lett. 107 (2018) 398.

[109] E. Pian et al., Nat 551 (2017) 67.

[110] R. Poggiani, in Frontier Research in Astrophysics - III, PoS(FRAPWS2018)013.

[111] D. Pooley et al., ApJL 859 (2018) L23.

[112] A. S. Pozanenko et al., ApJ 852 (2018) L30.

[113] L. Resmi et al., ApJ 867 (2018) 57.

[114] A. G. Riess et al., ApJ 855 (2018) 136.

[115] J. R. Ruan et al., ApJ 853 (2018) L4.

[116] O. S. Salafia et al., MNRAS 474 (2018) L7.

[117] R. H. Sanders, IJMPD 27 (2018) 1847027. 
[118] V. Savchenko et al., ApJ 848 (2017) L15.

[119] B. F. Schutz, Nat 323 (1986) 310.

[120] B. J. Shappee et al., Sci 358 (2017) 1574.

[121] M. R. Siebert et al., ApJL 848 (2017) L26.

[122] S. J. Smartt et al., Nat 551 (2017) 75.

[123] M. Soares-Santos et al., ApJL 848 (2017) L16.

[124] S. Sugita et al., PASJ 70 (2018) 81.

[125] N. R. Tanvir et al., Nat 500 (2013) 547.

[126] N. R. Tanvir et al., ApJL 848 (2017) L27.

[127] N. Tominaga et al., PASJ 70 (2018) 28.

[128] E. Troja et al., Nat 551 (2017) 71.

[129] E. Troja et al., MNRAS 478 (2018) L18.

[130] E. Troja et al., MNRAS 489 (2019) 1919.

[131] E. Troja et al., arXiv:2006.01150.

[132] Y. Utsumi et al., PASJ 69 (2017) 101.

[133] S. Valenti et al., ApJL 848 (2017) L24.

[134] F. Verrecchia et al., ApJ 850 (2017) L27.

[135] V. A. Villar et al., ApJL 851 (2017) L21.

[136] V. A. Villar et al., ApJ 862 (2018) L11.

[137] S. Yang et al., ApJ 875 (2019) 59. 